\title{
Pharmacogenomics of anti-TNF response in psoriasis, where are we?
}

\author{
"...the identification of biomarkers for anti-TNF response has become \\ one of the main objectives of translational research in psoriasis."
}

First draft submitted: 10 November 2015; Accepted for publication: 10 November 2015; Published online: 12 February 2016

Keywords: consortium • genome-wide association study • immune-mediated inflammatory diseases $\bullet$ pharmacogenetics $\bullet$ psoriasis

\section{Anti-TNF therapies: a breakthrough in the management of severe psoriasis}

Psoriasis is a chronic immune-mediated inflammatory disease that affects approximately $2 \%$ of the world's population. It is characterized by the immune infiltration in the epidermis and the hyperproliferation of keratinocytes. Despite not being a lifethreatening disease, it can have a negative impact in the quality of life of many patients. Approximately a third of psoriasis patients have a moderate-to-severe form of the disease, which has a marked effect on their psychological well-being [1]. Fortunately, active research in the biotechnology sector have led to the development of new therapies - mainly biological therapies - that have shown to be highly efficient in reducing the proinflammatory process in psoriasis.

Anti-TNF agents are the main example of new, highly-successful therapies. Drugs aimed at blocking TNF cytokine were initially developed for the management of rheumatoid arthritis, a prevalent immunemediated inflammatory disease affecting the synovial joints [2]. In rheumatoid arthritis, high levels of TNF are expressed in the inflamed synovial tissue, thereby becoming a natural drug target. While experimental evidence of increased TNF production in psoriatic lesions had been also reported, this proinflammatory cytokine was not initially considered to be a central cytokine in psoriasis etiology [3]. However, the evidence that anti-TNF agents efficiently reduced skin inflammation in psoriatic arthritis, a disease that combines chronic inflammation in joints and skin, prompted the evaluation and approval of this group of biological therapies for the management of psoriasis [4].

In this last decade, anti-TNF agents have increasingly shown that they are highly efficacious treatments in psoriasis patients with more severe symptoms. As evidence has been accrued, dermatologists have felt more confident in the incorporation of these drugs into their daily clinical practice [5]. This experience has also shown that despite being a useful therapy for many patients, there is also a substantial group of patients that do not show significant clinical improvement with anti-TNF agents [6]. Like other immune-mediated inflammatory diseases, psoriasis shows also a heterogeneous response to TNF blockade. Given the high costs of these therapies, the potential for adverse side effects and, also, the increasing availability of alternative therapeutic strategies, the identification of biomarkers for anti-TNF response has become one of the main objectives of translational research in psoriasis [7].

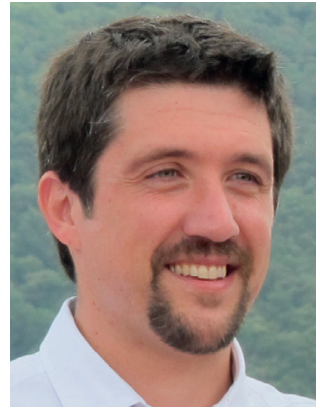

Antonio Julià

Rheumatology Research Group, Vall d'Hebron Research Institute, Barcelona, Spain

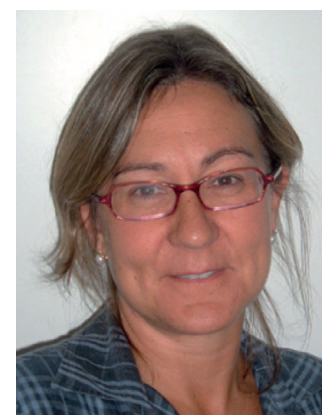

Sara Marsal

Author for correspondence:

Rheumatology Research Group, Vall d'Hebron Research Institute, Barcelona, Spain

sara.marsal@vhir.org 


\section{Search for genetic variants associated with} anti-TNF treatment response in psoriasis

Genetic variation is an appealing source of biomarkers for treatment response prediction since it is highly stable and it is easy to obtain. Also, advances in DNA genotyping and sequencing are making these technologies widely accessible to clinical diagnostic laboratories [8]. Together, these advantages position genetic variation at the forefront of useful individual information to personalize medicine. However, in order to be sufficiently informative to help in clinical decisionmaking, genetic variants must show relatively strong effects. Importantly, the predictive ability of a genetic variant (or any other potential biomarker) should not be confused with the statistical significance of the association with treatment response [9]. In many pharmacogenetic studies, the association of genetic variants with treatment response could be statistically significant but individually carry little predictive information. Importantly, if the final objective is to incorporate such variants in clinical decision-making, these biomarkers should surpass robust evaluation frameworks like randomized clinical trials [10].

\section{"Genome-wide association studies will soon be carried out and will show the extent at which common variation is important in the response to anti-TNF therapy in psoriasis."}

To date, most pharmacogenetic studies of anti-TNF response in psoriasis have been based on the candidategene approach [7]. In this approach, biological knowledge is used to hypothesize the association of a particular gene with the disease or any other trait. With this method, suggestive associations between genes from TNF pathway and other inflammatory pathways with anti-TNF response have been shown [6,11]. Like any other gene-to-trait association, however, these genetic associations need to be validated in independent patient cohorts from different populations. To date, this consistent replication has not yet been achieved in candidate genes for anti-TNF response in psoriasis. Important conditioning factors for the success of these validation studies are the use of patient cohorts of sufficient sample size as well as a strict control of the phenotypic diversity present in the patient samples [12].

Given the constraints on biological sample and clinical information collection as well as the need for larger cohorts of patients in translational research, it is becoming more and more common the establishment of large biomedical consortia. In psoriasis, two such examples are the Immune-Mediated Inflammatory Disease (IMID) consortium [13] and the Psoriasis Stratification to Optimize Relevant Therapy
(PSORT) consortium [14]. The IMID consortium is a network of clinical specialists and biomedical researchers in immune-mediated inflammatory diseases from $>90$ clinical departments from Spain. One of the main objectives of the IMID consortium is the advancement of translational medicine in psoriasis through the use of large-scale molecular analysis technologies. To date, this biomedical network has enabled the characterization of new genetic aspects in psoriasis including response to anti-TNF therapy $[13,15]$. The PSORT consortium is a public-private partnership including dermatology investigators from the UK as well as companies from the pharmaceutical and biotechnological sectors [14]. The main objective of the PSORT is to identify new biomarkers for treatment personalization in psoriasis through the use of highthroughput genomic analysis approaches. National and international consortia, like these two examples, are going to be essential to identify relevant aspects of psoriasis molecular features, including the genetic predisposition to respond to anti-TNF therapies.

The candidate-gene approach is subject to the particular knowledge of the biological processes taking place in the disease which is, in turn, relatively limited. In the last decade, however, a more powerful and less subjective approach has appeared in the form of genome-wide association studies (GWAS) [12]. In GWAS, a large number of markers across the genome are genotyped per individual. This strategy has the advantage that does not require a specific a priori hypothesis as to what gene or genomic region is associated with the response to treatment. GWAS were triggered by the combination of the microarray technology that had been already in use for several years for the genome-wide analysis of gene expression, together with the acquired knowledge of the variation patterns in the human genome [16]. This new analysis approach has the potential to identify new genes and biological pathways associated that would not otherwise have been linked to drug response. Also, an important aspect to bear in mind is that, compared with disease risk, the responses to drugs like anti-TNF agents are not traits that have been subjected to selective pressure. Therefore, the genetic variants associated to drug response have the potential to have very large effect sizes. To date, there is yet no GWAS for anti-TNF response in psoriasis but it is very likely that in the near future new risk locus for anti-TNF response will be identified through this approach.

\section{Pharmacogenetic variation associated to anti-TNF response might be shared}

From a statistical perspective, for a genetic variant to be considered a true risk factor for a disease or trait, 
it must withstand successive replication in independent cohorts. However, failing to replicate not necessarily implies that the genetic variation is a false positive, since other factors like sample size, effect size of the variation and the underlying risk model impact on the statistical power to replicate an association. With the advent of GWAS, however, a consensual genome-wide level of significance has been reached [12]. This threshold (commonly recognized as a $p<5 e-8)$ represents the strongest evidence for association with a trait.

So far, no pharmacogenetic variant has been associated at this level of statistical significance with psoriasis anti-TNF treatment in psoriasis. Recently, however, a variation at PDE3A-SLCO1C1 locus on chromosome 12 has been associated with the response to anti-TNF treatment in rheumatoid arthritis [17]. After combining the evidence from patients from two different European populations (Denmark and Spain), PDE3A-SLCO1C1 reached a genome-wide level of significance. Given this strong statistical evidence and the fact that the same group of antiTNF agents are used with relatively similar response rates, this pharmacogenetic variant was subsequently tested in the response to anti-TNF therapies in psoriasis [15] and psoriatic arthritis [18]. In both studies, this genomic region showed a significant association with the clinical response to anti-TNF treatment. The discovery of PDE3A-SLCO1C1 pharmacogenetic association in three different diseases suggests that immune-mediated inflammatory diseases not only share a common genetic risk basis, but they also share common genetic variation associated with the response to anti-TNF therapy. These results demonstrate that the characterization of genetic variation associated with treatment response can also contribute to the understanding of the specific biological mech-

\section{References}

1 Boehncke WH, Schon MP. Psoriasis. Lancet 386(9997), 983-994 (2015).

2 Feldmann M. Development of anti-TNF therapy for rheumatoid arthritis. Nat. Rev. Immunol. 2(5), 364-371 (2002).

3 Lebwohl M. Biologics for psoriasis: a translational research success story. J. Invest. Dermatol. 135(5), 1205-1207 (2015).

4 Leonardi CL, Powers JL, Matheson RT et al. Etanercept as monotherapy in patients with psoriasis. N. Engl. J. Med. 349(21), 2014-2022 (2003).

5 Ferrándiz C, Carrascosa JM, Boada A. A new era in the management of psoriasis? The biologics: facts and controversies. Clin. Dermatol. 28(1), 81-87 (2010). anisms that are relevant in autoimmune diseases like psoriasis.

\section{Future perspective}

There is still much to investigate and learn in the pharmacogenetics of psoriasis. GWAS will soon be carried out and will show the extent at which common variation is important in the response to anti-TNF therapy in psoriasis. Based on the results from other pharmacogenomic GWAS on other common diseases, it is probable that genetic variation, by itself, will not be sufficiently informative to be used in the clinical practice. Instead, it is more likely that in the clinical practice of the future, the pharmacogenomic profile of a patient with moderate-to-severe psoriasis will be integrated with other sources of molecular, clinical and epidemiological information in order to help dermatologists to make the best decision and, ultimately, have a significant impact in the quality of life of these patients. To reach this ambitious objective, the increasing presence of public and private biomedical research consortia will be essential.

\section{Acknowledgements}

The authors thank all the clinical researchers from the Immune-Mediated Inflammatory Disease Consortium for participation.

\section{Financial \& competing interests disclosure}

This work was supported by the Spanish Ministry of Economy and Competitiveness grants (PI12/01362 and IPT-0100002010-36). The authors have no other relevant affiliations or financial involvement with any organization or entity with a financial interest in or financial conflict with the subject matter or materials discussed in the manuscript apart from those disclosed.

No writing assistance was utilized in the production of this manuscript.

6 Prieto-Perez R, Cabaleiro T, Dauden E, Abad-Santos F. Gene polymorphisms that can predict response to anti-TNF therapy in patients with psoriasis and related autoimmune diseases. Pharmacogenomics J. 13(4), 297-305 (2013).

7 O'Rielly DD, Rahman P. Pharmacogenetics of psoriasis. Pharmacogenomics 12(1), 87-101 (2011).

8 Willis JC, Lord GM. Immune biomarkers: the promises and pitfalls of personalized medicine. Nat. Rev. Immunol. 15(5), 323-329 (2015).

9 Dudbridge F. Power and predictive accuracy of polygenic risk scores. PLoS Genet. 9(3), e1003348 (2013).

10 Ioannidis J, Khoury MJ. Are randomized trials obsolete or more important than ever in the genomic era. Genome Med. 5(4), 32 (2013).

11 Prieto-Pérez R, Solano-López G, Cabaleiro T et al. The polymorphism rs 763780 in the $I L-17 F$ gene is associated 
with response to biological drugs in patients with psoriasis. Pharmacogenomics 16(15), 1723-1731 (2015).

12 Mccarthy MI, Abecasis GR, Cardon LR et al. Genome-wide association studies for complex traits: consensus, uncertainty and challenges. Nat. Rev. Genet. 9(5), 356-369 (2008).

13 Julia A, Tortosa R, Hernanz JM et al. Risk variants for psoriasis vulgaris in a large case-control collection and association with clinical subphenotypes. Hum. Mol. Genet. 21(20), 4549-4557 (2012).

14 Reynolds N. One hundred and twenty-five years and counting: into an era of systems dermatology. Br. J. Dermatol. 171(6), 1279-1281 (2014).

15 Julia A, Ferrandiz C, Dauden E et al. Association of the PDE3A-SLCO1C1 locus with the response to anti-TNF agents in psoriasis. Pharmacogenomics J. 15(4), 322-325 (2015)

16 International HapMap Consortium. The International HapMap Project. Nature 426(6968), 789-796 (2003).

17 Acosta-Colman I, Palau N, Tornero J et al. GWAS replication study confirms the association of $P D E 3 A-$ SLCO1C1 with anti-TNF therapy response in rheumatoid arthritis. Pharmacogenomics 14(7), 727-734 (2013).

18 Julia A, Rodriguez J, Fernandez-Sueiro JL et al. PDE3A-SLCO1C1 locus is associated with response to anti-tumor necrosis factor therapy in psoriatic arthritis. Pharmacogenomics 15(14), 1763-1769 (2014). 